\title{
Síndrome de Bartter neonatal en un paciente prematuro. Reporte de caso y revisión narrativa de la bibliografía
}

\author{
Neonatal Bartter Syndrome in a premature patient. \\ Literature review
}

Eduardo Augusto Gálvez-Cuitiva, Jennifer Rojas-Muriel

\begin{abstract}
Resumen
ANTECDENTES: El síndrome de Bartter es una tubulopatía que afecta el transporte hidroelectrolítico; su prevalencia es de un caso por cada millón de habitantes. Se han descrito cinco subtipos de característica autosómica recesiva y un subtipo autosómico dominante. Del total de estos, cuatro subtipos son de presentación antenatal, con característica sobresaliente de poliuria que genera polihidramnios, prematurez y, en el periodo neonatal, alteraciones hidroelectrolíticas que llevan a lesión renal y alteraciones neurológicas.

CASO CLíNICO: Recién nacido, producto del segundo embarazo, madre de 22 años que inició con aumento importante del perímetro abdominal hacia las 12 semanas de gestación. Se optó por una conducta expectante hasta la semana 23 en la que se estableció el diagnóstico de polihidramnios severo, sin reporte de alteración estructural fetal. Se sospechó síndrome de Bartter que se confirmó con los estudios correspondientes. Se trató con ibuprofeno y la respuesta fue satisfactoria

CONCLUSIÓN: La ausencia de identificación de esta enfermedad, junto con el inadecuado tratamiento hidroelectrolítico de estos pacientes conducen a cuadros de deshidratación y alteraciones hidroelectrolíticas severas que pueden desencadenar insuficiencia renal, algunos subtipos de sordera neurosensorial, alteraciones en el neurodesarrollo y muerte.
\end{abstract}

PALABRAS CLAVE: Síndrome de Bartter; antenatal; embarazo; polihidramnios; poliuria; ibuprofeno.

\begin{abstract}
BACKGROUND: Bartter syndrome is a tubulopathy affecting water-electrolyte transport; its prevalence is one case per million population. Five autosomal recessive subtypes and one autosomal dominant subtype have been described. Of these, four subtypes are of antenatal presentation, with an outstanding characteristic of polyuria that generates polyhydramnios, prematurity and, in the neonatal period, hydroelectrolytic alterations that lead to renal lesions and neurological alterations.

CLINICAL CASE: Newborn, product of the second pregnancy, 22-year-old mother who started with a significant increase in abdominal perimeter around 12 weeks of pregnancy. Expectant management was chosen until the 23rd week, when the diagnosis of severe polyhydramnios was established, with no report of fetal structural alteration. Bartter's syndrome was suspected and confirmed with the corresponding studies. She was treated with ibuprofen and the response was satisfactory.

CONCLUSION: The lack of identification of this disease, together with the inadequate hydroelectrolytic treatment of these patients leads to dehydration and severe hydroelectrolytic alterations that can trigger renal failure, some subtypes of sensorineural
\end{abstract} deafness, neurodevelopmental alterations and death.

KEYWORDS: Bartter syndrome; Antenatal; Pregnancy; Polyhydramnios; Polyuria. Polyuria; Ibuprofen.
Instituto Materno Infantil. Sub Red Centro Oriente Bogotá. Universidad Nacional de Colombia.

Recibido: 30 de mayo 2019

Aceptado: 23 de noviembre 2020

Correspondencia

Eduardo Augusto Gálvez-Cuitiva egalvezcuitiva@yahoo.com.mx

Este artículo debe citarse como: GálvezCuitiva EA, Rojas-Muriel J. Síndrome de Bartter neonatal en un paciente prematuro. Reporte de caso y revisión narrativa de la bibliografía. Acta Pediatr Méx 2021; 42 (3): 121-27. 


\section{ANTECEDENTES}

El síndrome de Bartter es una tubulopatía que afecta el asa de Henle y el túbulo contorneado distal; fue descrita en 1962 por Frederic Bartter y sus colaboradores. Hasta el momento se han descrito diferentes subtipos, que se distinguen uno de otro por el canal iónico afectado. ${ }^{1}$ Se han definido las formas neonatales tipos I, II, IV (a y b) y $V^{1,2}$ aunque debe tenerse en cuenta que a pesar de que ésta es la presentación habitual de esos subtipos, se han reportado casos con presentación variable, con síndrome de Bartter tipo I, II o IV de inicio tardío y formas muy tempranas en pacientes diagnosticados con síndrome de Bartter tipo III. ${ }^{2}$

Estos tipos de tubulopatías pueden sospecharse desde el periodo fetal y en la vida neonatal al confirmarse con un diagnóstico oportuno, a través de la cuantificación de las concentraciones de electrolitos, renina y aldosterona, entre otros. ${ }^{3}$ Sus manifestaciones están dadas por alteraciones en los canales transportadores de sodio, potasio, cloro, calcio y magnesio. ${ }^{3}$

La conducta médica inicial incluye el adecuado control hidroelectrolítico y, con base en la fisiopatología de la enfermedad, el tratamiento convencional con antiinflamatorios no esteroideos (AINES); la indometacina es la más prescrita. $^{3}$

Se describe el caso de un paciente prematuro, con manifestaciones clínicas desde el periodo prenatal, en quien se llevó a cabo el procedimiento conducente para establecer el diagnóstico y tratamiento en el contexto de un síndrome de Bartter antenatal, tratado con ibuprofeno y desenlace favorable.

\section{CASO CLÍNICO}

Recién nacido, producto del segundo embarazo, madre de 22 años que inició con aumento importante del perímetro abdominal hacia las 12 semanas de embarazo. Se optó por una conducta expectante hasta la semana 23 en la que se estableció el diagnóstico de polihidramnios severo, sin reporte de alteración estructural fetal. Requirió amniocentesis reductora y se indicó tratamiento con indometacina durante una semana. A las 25 semanas acudió a un hospital de tercer nivel de atención en donde se realizaron: antígeno de superficie de hepatitis B, prueba no treponémica (VDRL), anticuerpos IgM para citomegalovirus, rubéola y toxoplasma y pruebas rápidas para detección de $\mathrm{VIH}$ que resultaron negativas.

En la ecografía obstétrica se corroboró el polihidramnios severo con índice de líquido amniótico de $400 \mathrm{~mL}$; recibió esquema de corticoide antenatal con betametasona y, además, sulfato de magnesio. A las 26 semanas inició con trabajo de parto espontáneo por lo que se decidió finalizar el embarazo mediante cesárea.

El nacimiento fue en presentación cefálica, con abundante líquido amniótico, claro, no fétido, con esfuerzo respiratorio deficiente, adaptación inducida, pinzamiento al tercer minuto de nacido, APGAR 4-7-7 (al minuto, a los 5 y 10 minutos respectivamente), intubación orotraqueal y posterior dosis de surfactante exógeno.

Medidas antropométricas: peso $1000 \mathrm{~g}$ (percentil 90 de Fenton), talla: $38 \mathrm{~cm}$ (percentil 97 de Fenton). Se trasladó a la unidad de cuidados intensivos neonatales en donde se inició la nutrición parenteral, citrato de cafeína y lactobacilos reuteri de acuerdo con el protocolo institucional. Al examen físico resaltó la hipertricosis frontal, edema de cuero cabelludo generalizado, cara triangular, abdomen distendido con onda ascítica positiva y edema bimaleolar simétrico en las extremidades inferiores.

A partir de las primeras 12 horas de vida extrauterina tuvo gastos urinarios elevados, de 6.54 
$\mathrm{cc} / \mathrm{kg} / \mathrm{h}$ durante las primeras 12 horas de vida; $7.6 \mathrm{cc} / \mathrm{kg} / \mathrm{h}, 6.19 \mathrm{cc} / \mathrm{kg} / \mathrm{h}$ al segundo y tercer día de vida, respectivamente. Al tercer día de vida requirió aporte hídrico de 160 cc/kg/día, sin inicio de vía enteral durante ese tiempo por drenaje hemático fresco a través de sonda orogástrica por la que se administró la vitamina K complementaria. La concentración de electrolitos séricos del segundo día de vida fue de: $163.7 \mathrm{mEq} / \mathrm{L}$, potasio: $4.55 \mathrm{mEq} / \mathrm{L}$, calcio colorimétrico: 5.79 $\mathrm{mEq} / \mathrm{L}$, cloro: $118 \mathrm{mEq} / \mathrm{L}$, creatinina: $1.05 \mathrm{mg} /$ $\mathrm{dL}$, nitrógeno ureico sérico $(\mathrm{BUN}): 45 \mathrm{mg} / \mathrm{dL}$. Se corrigieron los aportes de sodio, calcio y se disminuyó el aporte de proteínas de 3.5 a 2 g/ kg/día en la nutrición parenteral.

El ecocardiograma reportó: conducto arterioso con repercusión ecocardiográfica. Se trató con 12 dosis de $15 \mathrm{mg} / \mathrm{kg}$ de paracetamol, con cierre exitoso observado en la ecografía transfontanelar al tercer día de vida, con hemorragia de la matriz germinal izquierda grado I, con control ecográfico a los 15 días con hemorragia cohibida.

Al séptimo día de vida persistió la poliuria, con aporte hídrico de $180 \mathrm{~mL} / \mathrm{kg} /$ día, pérdida del 15\% del peso (pesaba $850 \mathrm{~g}$ ). A los 8 días de vida el aporte hídrico fue de $180 \mathrm{cc} / \mathrm{kg} /$ día, con exámenes de control de sodio: $130.9 \mathrm{mEq} / \mathrm{L}$, potasio: $4.45 \mathrm{mEq} / \mathrm{L}$, calcio: $7.04 \mathrm{mEq} / \mathrm{L}$, cloro: $89.9 \mathrm{mEq} / \mathrm{L}$, fósforo: $8.75 \mathrm{mEq} / \mathrm{L}$, magnesio: $2.1 \mathrm{mEq} / \mathrm{L}, \mathrm{BUN}: 46 \mathrm{mg}$, creatinina: $1.1 \mathrm{mg}$, filtración glomerular de $11.4 \mathrm{~mL} / \mathrm{min} / 1.73 \mathrm{~m}^{2}$. Recibió electrolitos por nutrición parenteral con aporte $12 \mathrm{meq} / \mathrm{kg} /$ día de sodio, $6 \mathrm{meq} / \mathrm{kg} /$ día de potasio, $150 \mathrm{mg} / \mathrm{kg} /$ día de calcio. Ante el desequilibrio hidroelectrolítico persistente con aportes altos de sodio y calcio, se consideró probable tubulopatía.

Las concentraciones de electrolitos urinarios, con fracción excretada de sodio (FENa) se reportaron en $2.42 \%$, fracción excretada de potasio (FEK) de $28.1 \%$, fracción excretada de calcio (FECa) de $1.35 \%$, calciuria en orina de 24 horas de $10.26 \mathrm{mg} / \mathrm{m}^{2} / \mathrm{h}$ (valores normales para población pediátrica: FeNa menos de $1 \%$, FeK de 2 a 10\%, FeCa menor a $0.08 \%$ y Ca en 24 horas menor de $4 \mathrm{mg} / \mathrm{m}^{2} / \mathrm{h}$ ). Las concentraciones de renina y aldosterona se reportaron elevadas. La ecografía renal y de vías urinarias evidenció la nefrocalcinosis. Con lo anterior se estableció el diagnóstico de síndrome de Bartter e inició el tratamiento con $5 \mathrm{mg} / \mathrm{kg} /$ día de ibuprofeno, con ascenso progresivo de $2 \mathrm{mg} / \mathrm{kg} /$ día hasta lograr los valores normales de sodio, potasio y calcio. Se alcanzó la dosis de $10 \mathrm{mg} / \mathrm{kg} /$ día al décimo quinto día de vida, con adecuado control hidroelectrolítico, transición a la nutrición enteral total y sin requerimiento extra de sodio y potasio. Cuadro 1

\section{DISCUSIÓN}

En condiciones normales, el 99\% del sodio y el agua filtrados se reabsorben a lo largo de los túbulos renales. En el caso específico del sodio se reabsorbe en 70 a $80 \%$ en el túbulo proximal, 10 a $20 \%$ en la porción gruesa del ascendente del asa de Henle, 5 a $10 \%$ en el túbulo distal

Cuadro 1. Evolución de los análisis de laboratorio

\begin{tabular}{|c|c|c|c|c|c|c|c|c|}
\hline Día & Na & $\mathbf{K}$ & $\mathbf{C l}$ & $\mathbf{C a}$ & $\mathbf{P}$ & $\mathbf{M g}$ & $\mathbf{B U N}$ & $\begin{array}{c}\text { Crea- } \\
\text { tinina }\end{array}$ \\
\hline 3 & 163.7 & 4.55 & 5.79 & & 45 & 1.05 \\
\hline 4 & 164.7 & 3.82 & 6.67 & & 48.2 & 1.01 \\
\hline 5 & 155.3 & 4.22 & 105.1 & 7.58 & & 74 & 1.43 \\
\hline 6 & 130.9 & 4.45 & 89.9 & 7.04 & 8.75 & 46.5 & 1.17 \\
\hline 7 & 118.4 & 3.65 & 6.49 & & & \\
\hline 9 & 113.4 & 3.43 & 6.54 & & & \\
\hline 10 & 119 & 2.45 & 7.67 & & & \\
\hline 11 & 127 & 3.19 & 7.7 & & 4.9 & 0.29 \\
\hline 13 & 130.3 & 3.74 & 92 & 8.31 & 1.77 & & \\
\hline 17 & 129.2 & 2.8 & 9.9 & & & \\
\hline 18 & 134.2 & 5.29 & & & & \\
\hline 19 & 133.4 & 4.25 & 9.92 & & & \\
\hline 21 & 132.8 & 2.29 & 9.39 & & & \\
\hline 23 & 133 & 2.51 & 8.97 & &
\end{tabular}


y el 2 al 5\% restante en la nefrona sensible a aldosterona. El potasio se absorbe en un $20 \%$ en el asa de Henle. El síndrome de Bartter se centra en las alteraciones del asa ascendente de Henle y el túbulo distal. ${ }^{3,4,5}$

El asa ascendente cuenta con un cotransportador de sodio-potasio-2cloro (NKCC2) encargado de ingresar estos iones al interior de la célula para que, posteriormente, la bomba sodio-potasio ATPasa, ubicada en la membrana baso lateral, intercambie sodio hacia el torrente sanguíneo. El potasio tiene un transportador tipo ROMK que lo retorna al lumen tubular para permitir el funcionamiento del cotransportador NKCC2 $y$, a su vez, para generar un gradiente electroquímico que permite el paso de calcio y magnesio. ${ }^{6,7} \mathrm{En}$ la membrana basolateral (CLKa y CLKb) el cloro tiene dos canales de voltaje dependientes encargados de la conductancia del cloro al torrente sanguíneo. Para su activación, estos canales dependen de la existencia de la proteína barttina. ${ }^{6,7}$

Respecto al túbulo distal, éste cuenta con un cotransportador sodio-cloro sensible a tiazidas (NCCT). Funciona en conjunto con las bombas $\mathrm{Na} / \mathrm{K}$ ATPasa en la membrana basolateral y los canales rectificadores de potasio (Kir4.1) que facilitan el paso basolateral de potasio permitiéndole suficiente sustrato al cotransportador. En el túbulo distal hay canales para la absorción de calcio y magnesio que son independientes del gradiente transtubular de potasio, como ocurre en el asa de Henle. En la membrana basolateral su paso al torrente sanguíneo está dado por canales intercambiadores de calcio-sodio y magnesio-sodio. En el caso del calcio existen, además, canales transportadores específicos de calcio en la membrana basolateral. ${ }^{8,9}$

En la mácula densa se supervisa la concentración de sodio tubular, que se lleva a cabo mediante los mismos transportadores NKCC2, ROMK y los canales de cloro, que generan un cambio de tensión en la membrana basolateral y una movilización de calcio que origina las correspondientes cascadas de señalización intracelular. Esto se refleja en mecanismos que, por medio de la liberación de prostaglandina E2, ATP, adenosina y óxido nítrico, modulan la absorción glomerular y tubular de sodio. Así, el aumento de las concentraciones de potasio tubular y la activación de canales de calciovoltaje dependientes conducen a la liberación de prostaglandinas vasoconstrictoras de las arteriolas aferentes, lo que finalmente se ve reflejado en un aumento de liberación de renina. Respecto al sodio, al reducirse su transporte caen los volúmenes extracelulares y se estimula la liberación de renina. ${ }^{9}$

Gracias al desarrollo de técnicas de biología molecular se han logrado catalogar los diferentes tipos: el síndrome de Bartter tipo III, o Bartter clásico de manifestación tardía, mientras que los subtipos I, II, IV y $V$ se han descrito como formas de presentación neonatal. Los tipos I y II son de aparición antenatal severa y los tipos IV $y \vee$ se relacionan con sordera neurosensorial. ${ }^{10,11}$

\section{Cuadro 2}

\section{Síndrome de Bartter antenatal}

EL síndrome Bartter tipo I se distingue por la pérdida de la función del canal NKCC2, relacionado con el gen SLC12A1. Se estima que 1 de cada 360 individuos tiene un alelo causal de esta enfermedad. ${ }^{11}$ Desde el punto de vista clínico se inicia en el segundo trimestre del embarazo con poliuria fetal marcada, que en el útero genera polihidramnios que, en la totalidad de los casos, da lugar a parto prematuro asociado con retraso del crecimiento. En el líquido amniótico hay concentraciones elevadas de cloro mientras que las de sodio, potasio, calcio y prostaglandina $\mathrm{E}$ son normales. ${ }^{12,13}$

El síndrome de Bartter tipo II se origina por una alteración en la función del cotransportador ROMK1, cuya mutación genética es dada por 
Gálvez-Cuitiva EA, et al. Síndrome de Barter neonatal

Cuadro 2. Subtipos de síndrome de Bartter, mutaciones genéticas asociadas y asociaciones clínicas características²

\begin{tabular}{|c|c|c|c|c|c|}
\hline & Presentación & Proteína & Gen & OMIN & Clínica \\
\hline Tipo I & Antenatal & NKCC2 & SLC12A1 & 601678 & $\begin{array}{l}\text { Polihidramnios severo, hiponatremia, hipopotase- } \\
\text { mia, nefrocalcinosis }\end{array}$ \\
\hline Tipo II & Antenatal & ROMNK1 & KCNJ1 & 241200 & $\begin{array}{l}\text { Polihidramnios menos severo, hiponatremia, hi- } \\
\text { percalemia transitoria, nefrocalcinosis }\end{array}$ \\
\hline Tipo III & Tardía & CLC-Kb & CLCNKB & 607364 & $\begin{array}{l}\text { Alcalosis hipoclorémica, hipopotasemia, poliuria } \\
\text { variable }\end{array}$ \\
\hline $\begin{array}{l}\text { Tipo } \\
\text { IV a y b }\end{array}$ & Antenatal & $\begin{array}{l}\text { Barttina, CLC-Ka, } \\
\text { CLC-Kb }\end{array}$ & $\begin{array}{l}\text { BSND, CLCNKA, } \\
\text { CLCNKB }\end{array}$ & 602522 & $\begin{array}{l}\text { Polihidramnios, hipopotasemia, sordera neuro- } \\
\text { sensorial }\end{array}$ \\
\hline Tipo V & Variable & CASR & CASR & 300971 & $\begin{array}{l}\text { Hipocalemia, hiponatremia transitorios, polihi- } \\
\text { dramnios severo }\end{array}$ \\
\hline
\end{tabular}

una alteración en el gen KCNJ1. Uno de cada 670 individuos es portador de un alelo mutante para esta enfermedad. ${ }^{13} \mathrm{Al}$ igual que todas las formas de síndrome de Bartter neonatal, ésta se manifiesta con polihidramnios, de menor severidad que el tipo I $y$, por ende, parto no tan prematuro. El eje renina-angiotensinaaldosterona está conservado y lo que sucede es una insensibilidad a la vasopresina, que genera isostenuria, hipercalciuria e hipercalemia transitoria en los primeros días. Se manifiesta junto con acidosis metabólica secundaria, que disminuye en las primeras semanas y que más tarde configura la hipopotasemia característica, aunque leve, y la alcalosis metabólica, que la diferencia del hipoaldosteronismo o la hiperplasia suprarrenal. Al igual que en el síndrome de Bartter tipo I, la hipermagnesuria e hipomagnesemia no son comunes en virtud de la compensación en la absorción de magnesio en el túbulo distal. ${ }^{13}$

El síndrome Bartter tipo IV caracterizado por la alteración en la barttina o en los transportadores CLC-Ka y CLC-Kb configura el trastorno de pérdida de cloruro de sodio más severo. Desde el punto de vista clínico se manifiesta, al igual que los otros subtipos, con polihidramnios, prematurez, choque hipovolémico y lesión renal. ${ }^{13}$ Como factor diferenciador, la hipoacusia es característica de este subtipo, debida a la alteración en la secreción de endolinfa en el oído interno; en su composición requiere iones de potasio, transportados por los transportadores NKCC2, que se ve limitado por la ausencia de iones de cloro. ${ }^{13,14}$ En este subtipo tampoco hay pérdidas aumentadas de calcio en la orina, por lo que no hay nefrocalcinosis pero sí hipermagnesuria con hipomagnesemia consecuencia de la afectación en la absorción de magnesio en el asa de Henle y en el túbulo distal.

El síndrome de Bartter tipo $\mathrm{V}$, originado por una alteración en la proteína MAGED2 se asocia con mutación del gen que lleva este mismo nombre, con herencia autosómica recesiva y ligada a X. Esta proteína se ha relacionado con la inadecuada síntesis, funcionamiento y maduración de los transportadores NKCC2 y NCC que, en estados patológicos genera polihidramnios, parto prematuro y alteraciones hidroelectrolíticas. Estas alteraciones revierten espontáneamente entre las semanas 30 y 33. No es clara la razón del carácter transitorio de éste, hay hipótesis que señalan el aumento de la sensibilidad a la vasopresina como desencadenante de la subsecuente síntesis de los transportadores. También se propone que el aumento de las concentraciones de oxígeno en la vida posnatal aumenta su expresión. ${ }^{14}$

Ante la sospecha de síndrome de Bartter antenatal, el método diagnóstico de elección en el periodo antenatal es la amniocentesis con cuantificación de las concentraciones de electrolitos, prostaglandinas, renina, proteinas, ${ }^{15,16} \mathrm{y}$ 
para análisis de biología molecular de biopsia de vellosidades coriónicas, que es sensible desde etapas muy tempranas de la gestación, aunque implica mayores riesgos. ${ }^{16}$ En el periodo neonatal, las fracciones excretadas de sodio, potasio, cloro y calcio son elevadas y se asocian con alcalosis metabólica hipocalémica en los gases sanguíneos. Las concentraciones séricas de renina y aldosterona disminuidas se reflejan en altos niveles de sodio, potasio, cloro y calcio séricos. La hipercalciuria puede generar osteopenia, con concentraciones de PTH normales. ${ }^{16}$ Debe practicarse una ecografía renal para evidenciar o no nefrocalcinosis y análisis moleculares para determinar el tipo de síndrome y llevar a cabo la consejería a la familia. ${ }^{16,17}$

El tratamiento debe enfocarse en el adecuado control hidroelectrolítico. Deben indicarse inhibidores de la síntesis de prostaglandinas, como los antiinflamatorios no esteroideos. De estos, el más indicado es la indometacina, a dosis de 1 a $5 \mathrm{mg} / \mathrm{kg} / \mathrm{día},{ }^{17}$ acompañada de vigilancia estricta de los potenciales efectos adversos derivados de la lesión renal y perforación intestinal. También está descrita la indicación de 15 a $30 \mathrm{mg} / \mathrm{kg} /$ día de ibuprofeno, ${ }^{17}$ con desenlaces igualmente favorables. El tratamiento con AINES se asocia con disminución de las concentraciones de renina e incremento del potasio sérico, mejor poliuria e hipercalciuria pero sin revertir la nefrocalcinosis. ${ }^{18}$ En pacientes con hipopotasemia persistente se han observado efectos favorables a corto plazo con los inhibidores de la enzima corvertidora de angiotensina (IECA), a pesar del tratamiento con AINES y complementos con potasio, ${ }^{19}$ así como utilidad en pacientes con proteinuria y la indicación de ahorradores de potasio, con efectos transitorios por lo que su prescripción es motivo de controversia. ${ }^{19}$

El pronóstico a largo plazo de estos pacientes con diagnóstico y tratamiento oportuno es bueno. Por ello, las alteraciones clínicas son menos severas al superar el periodo neonatal; en algunos casos se consigue reducir, progresivamente, hasta suspender el tratamiento médico. ${ }^{19}$ En estos pacientes debe hacerse un seguimiento estricto hidroelectrolítico, del patrón de crecimiento y de la función renal.

\section{CONCLUSIÓN}

El síndrome de Bartter neonatal es una enfermedad de aparición temprana que, en todos los casos, se manifiesta con polihidramnios causado por la poliuria fetal, que persiste en el periodo neonatal. La ausencia de identificación de esta enfermedad, junto con el inadecuado tratamiento hidroelectrolítico, conduce a cuadros de deshidratación y alteraciones hidroelectrolíticas severas que pueden desencadenar insuficiencia renal, algunos subtipos de sordera neurosensorial, alteraciones en el neurodesarrollo y muerte. El tratamiento oportuno con AINES es decisivo para el pronóstico a largo plazo de estos pacientes. En el paciente del caso se obtuvo mejoría con ibuprofeno, sin las complicaciones reportadas con la indometacina, por lo que es una alternativa a valorar en este tipo de pacientes.

\section{REFERENCIAS}

1. Legrand A, Treard C, Roncelin I, Dreux S, et al. Prevalence of Novel MAGED2 Mutations in Antenatal Bartter Syndrome. CJASN 2018, 13 (2): 242-50. https://doi.org/10.2215/ CJN.05670517.

2. Kleta R. Salt-Losing Tubulopathies in Children : What's New , What's Controversial ? JASN 2018; 29 (3); 727-739. https://doi.org/10.1681/ASN.2017060600.

3. López Pérez JJ, Jaimes Martínez LF, Galvis Alvarado EF. Síndrome de Bartter. Reporte de un caso y revisión de la literatura. Revista Med 2011; 19 (2): 185-206. https://doi. org/10.18359/rmed.1280.

4. Shaer AJ. Inherited primary renal tubular hypokalemic alkalosis: a review of Gitelman and Bartter syndromes. Am J Med Sci 2001; 322 (6): 316-32. doi.10.1097/00000441200112000-00004. PMID: 11780689.

5. Waldegger S. Bartter, Gitelman, and Related Syndromes. Comprehensive Pediatr Nephrol. 2008. 451-59. 10.1016/ B978-0-323-04883-5.50035-0.

6. Bhat.Y, Ramesh Vinayaka, G Sreelakshmi, K. Antenatal Bartter Syndrome: A Review. Int J Pediatr 2012. https:// doi.org/10.1155/2012/857136. 
Gálvez-Cuitiva EA, et al. Síndrome de Barter neonatal

7. Flores F, Ojeda F, Calhoun DA. Bartter syndrome: presentation in an extremely premature neonate. J Perinatol 2013; 33: 661-62. https://doi.org/10.1038/jp.2013.13.

8. Fraga Rodríguez GM, Huertes Díaz B. Evaluación básica de la función renal en Pediatría. Protoc Diagn Terap Pediatr 2014; 1: 21-35.

9. Kastl JT. Renal function in the fetus and neonate. The creatinine enigma. Semin Fetal Neonatal Med 2017; 22 (2): 83-89. doi: 10.1016/j.siny.2016.12.002. Epub 2017 Jan 18. PMID: 28109705.

10. Seyberth HW, Weber S, Kömhoff M. Bartter's and Gitelman's syndrome. Curr Opin Pediatr 2017; 29 (2): 179-86. doi: 10.1097/MOP.0000000000000447. PMID: 27906863.

11. Ji W, Foo JN, O'Roak BJ, Zhao H, Larson MG, Simon DB, Newton-Cheh C, State MW, Levy D, Lifton RP. Rare independent mutations in renal salt handling genes contribute to blood pressure variation. Nat Genet 2008; 40 (5): 592-99. doi: 10.1038/ng.118. Epub 2008 Apr 6. PMID: 18391953; PMCID: PMC3766631.

12. Azzi A, Chehade $H$, Deschênes $G$. Neonates with Bartter syndrome have enormous fluid and sodium requirements. Acta Paediatrica 1992; 104 (7): e294-9. doi: 10.1111/ apa.12981.

13. Amirlak IKP. Dawson, Bartter syndrome: an overview. QJM Int J Med 2000; 93 (4): 207-15. https://doi.org/10.1093/ qjmed/93.4.207.
14. Roy A, Chakraborty D, Dogra S, Sengupta S. Neonatal Bartter Syndrome. J Nepal Paediatr Soc 2016; 36 (2): 204-7. https://doi.org/10.3126/jnps.v36i2.14818.

15. Laghmani K, Beck BB, Yang S-S, Seaayfan E, Wenzel A, Reusch B, et al. Polyhydramnios, transient antenatal Bartter's Syndrome, and MAGED2 mutations. N Engl J Med. 2016; 374 (19): 1853-63. doi.10.1056/NEJMoa1507629. Epub 2016 Apr 27. PMID: 27120771.

16. Garnier A, Dreux S, Vargas-Poussou R. Oury JF, Benachi $A$, Deschênes $G$, et al. Bartter syndrome prenatal diagnosis based on amniotic fluid biochemical analysis. Pediatr Res 2010; 67: 300-3. https://doi.org/10.1203/ PDR.0b013e3181ca038d.

17. Hegde D, Mondkar J, Abdagire N. Neonatal bartter syndrome in an extremely low birth weight baby. Saudi J Kidney Dis Transplant 2017; 28 (5): 1162-64. PMID 28937079.

18. Yang $X$, Zhang $G$, Wang $M$, Yang $H$, Li Q. Bartter Syndrome Type 3: Phenotype-genotype correlation and favorable response to ibuprofen. Front Pediatr 2018; 6: 153. doi: 10.3389/fped.2018.00153. PMID: 29900164; PMCID: PMC5989644.

19. Seyberth HW, Schlingmann KP. Bartter- and Gitelman-like syndromes: salt-losing tubulopathies with loop or DCT defects. Pediatr Nephrol 2011; 26 (10): 1789-802. doi: 10.1007/s00467-011-1871-4. Epub 2011 Apr 19. PMID: 21503667; PMCID: PMC3163795. 